\title{
Seasonal cycle and temperature dependence of pinene oxidation products, dicarboxylic acids and nitrophenols in fine and coarse air particulate matter
}

\author{
Y. Y. Zhang ${ }^{1}$, L. Müller ${ }^{2}$, R. Winterhalter ${ }^{1}$, G. K. Moortgat ${ }^{1}$, T. Hoffmann ${ }^{2}$, and U. Pöschl ${ }^{1}$ \\ ${ }^{1}$ Max Planck Institute for Chemistry, P.O. Box 3060, 55020 Mainz, Germany \\ ${ }^{2}$ Institute of Inorganic and Analytical Chemistry, Johannes Gutenberg Univ., Duesbergweg 10-14, 55128 Mainz, Germany
}

Received: 26 April 2010 - Published in Atmos. Chem. Phys. Discuss.: 25 May 2010

Revised: 26 July 2010 - Accepted: 27 July 2010 - Published: 25 August 2010

\begin{abstract}
Filter samples of fine and coarse air particulate matter (PM) collected over a period of one year in central Europe (Mainz, Germany) were analyzed for watersoluble organic compounds (WSOCs), including the $\alpha$ - and $\beta$-pinene oxidation products pinic acid, pinonic acid and 3methyl-1,2,3-butanetricarboxylic acid (3-MBTCA), as well as a variety of dicarboxylic acids and nitrophenols. Seasonal variations and other characteristic features in fine, coarse, and total PM (TSP) are discussed with regard to aerosol sources and sinks in comparison to data from other studies and regions. The ratios of adipic acid and phthalic acid to azelaic acid indicate that the investigated aerosol samples were mainly influenced by biogenic sources. A strong Arrhenius-type correlation was found between the 3-MBTCA concentration and inverse temperature $\left(R^{2}=\right.$ $0.79, n=52, E_{\mathrm{a}}=126 \pm 10 \mathrm{~kJ} \mathrm{~mol}^{-1}$, temperature range $275-$ $300 \mathrm{~K})$. Model calculations suggest that the temperature dependence observed for 3-MBTCA can be explained by enhanced photochemical production due to an increase of hydroxyl radical $(\mathrm{OH})$ concentration with increasing temperature, whereas the influence of gas-particle partitioning appears to play a minor role. The results indicate that the $\mathrm{OH}-$ initiated oxidation of pinonic acid is the rate-limiting step in the formation of 3-MBTCA, and that 3-MBTCA may be a suitable tracer for the chemical aging of biogenic secondary organic aerosol (SOA) by $\mathrm{OH}$ radicals. An Arrhenius-type temperature dependence was also observed for the concentra-
\end{abstract}

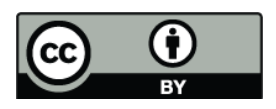

Correspondence to: Y. Y. Zhang (y.zhang@mpic.de) tion of pinic acid $\left(R^{2}=0.60, n=56, E_{\mathrm{a}}=84 \pm 9 \mathrm{~kJ} \mathrm{~mol}^{-1}\right)$; it can be tentatively explained by the temperature dependence of biogenic pinene emission as the rate-limiting step of pinic acid formation.

\section{Introduction}

Water-soluble organic compounds (WSOCs) are major components of atmospheric aerosols, accounting for up to $\sim 50 \%$ of the organic fraction of particulate matter. They can influence the optical properties and hygroscopicity of aerosol particles and their effects on climate. WSOCs originate from primary emissions like fossil fuel combustion and biomass burning as well as from secondary formation in the atmosphere, i.e., photooxidation of anthropogenic or biogenic precursors (Pöschl, 2005).

Organic acids are a prominent group of WSOCs and were found in urban, rural, marine and polar aerosols in various regions around the world as detailed below (Kawamura and Ikushima, 1993; Kawamura et al., 1996b; Kawamura and Usukura, 1993). This group includes monocarboxylic acids, dicarboxylic acids, oxocarboxylic acids and tricarboxylic acids. The total dicarboxylic acids account for 0.3 $11 \%$ of WSOC (Pavuluri et al., 2010 and references therein) and oxalic acid, malonic acid and succinic acid $\left(\mathrm{C}_{2}-\mathrm{C}_{4}\right)$ are the most abundant species in the dicarboxylic acid group (Kawamura and Ikushima, 1993). Dicarboxylic acids originate from a wide range of sources. Primary sources include motor exhaust (Kawamura and Kaplan, 1987), biomass combustion (Kundu et al., 2010; Lefer et al., 1994; Legrand

Published by Copernicus Publications on behalf of the European Geosciences Union. 
and DeAngelis, 1996; Narukawa et al., 1999) and oceanic emissions (Mochida et al., 2003b). Secondary formation sources are the photooxidation of unsaturated fatty aids and cyclic alkenes (Hatakeyama et al., 1987; Kawamura et al., 1996a). The photooxidation of biogenic hydrocarbons is a major source of secondary organic aerosol (SOA) (Hoffmann et al., 1997), and SOA formation by oxidation of pinene and other terpenes has been studied extensively (Hallquist et al., 2009). Pinic acid as a dicarboxylic acid and pinonic acid as an oxocarboxylic acid are major products of the ozonolysis or $\mathrm{OH}$-initiated oxidation of pinene (here and below pinene stands for both $\alpha$ - and $\beta$-pinene) (Atkinson and Arey, 2003; Hatakeyama et al., 1989, 1991; Yu et al., 1999a). Further reaction of the initial oxidation products of pinene leads to highly oxidized, acyclic, polar compounds (3hydroxyglutaric acid and 3-methyl-1,2,3-butanetricarboxylic acid, 3-MBTCA) (Szmigielski et al., 2007; Kourtchev et al., 2009; Jaoui et al., 2005). 3-MBTCA is formed by OH-initiated oxidation of cis-pinonic acid (Szmigielski et al., 2007) and was first detected in aerosol samples from Amazonia and Belgium (Kubatova et al., 2000, 2002). Another prominent group of WSOCs are nitrophenols like 2nitrophenol and 4-nitrophenol, which have been found in urban and rural aerosol, rainwater and snow samples. They originate from primary sources including motor vehicle exhaust (Nojima et al., 1983; Tremp et al., 1993), coal and wood combustion, as well as decomposition and hydrolysis reactions of herbicides and insecticides (Shafer and Schonherr, 1985). Moreover, they are formed by nitration of phenol and the transformation of benzaldehyde under photochemical smog conditions (Grosjean, 1985; Luttke and Levsen, 1997; Luttke et al., 1997). Road traffic is considered to be the main source of nitrophenols.

Nitrophenols are usually analyzed by gas chromatography coupled with a mass spectrometer (GC/MS), with an electron capture detector (GC-ECD), or with a nitrogen phosphorous detector (GC-NPD) (Herterich, 1991; Leuenberger et al., 1985; Luttke et al., 1997). Belloli et al. (1999) used high performance liquid chromatography coupled with a diode array detector (HPLC-DAD) for nitrophenol detection. Most studies of carboxylic acids used GC/MS after derivatization, i.e., conversion of the acids into methyl- or butylesters (Kawamura and Ikushima, 1993). Römpp et al. (2006) and Warnke et al. (2006) applied high performance liquid chromatography coupled with a mass spectrometer (HPLC/MS) for the analysis of organic acids in aerosol samples.

In this study, we apply HPLC/MS for the analysis of nitrophenols as well as carboxylic acids (aliphatic $\mathrm{C}_{5}-\mathrm{C}_{16}$ dicarboxylic acids, phthalic, pinic and pinonic acid, 3-MBTCA) in a large set of fine and coarse aerosol particle samples collected over a period of one year in central Europe (Mainz, Germany, June 2006 to May 2007).

\section{Methods}

\subsection{Collection of filter samples}

Aerosol samples were collected on glass fiber filters (Pall Corporation, Type A/A, 102-mm diameter) over a period of one year in Mainz, Germany (130 m a.s.l., June 2006-May 2007). The sampling station was positioned on a mast at the top of the Max Planck Institute for Chemistry (MPIC, $\sim 5 \mathrm{~m}$ above the flat roof of the 3 -story building) on the campus of the University of Mainz $\left(49^{\circ} 59^{\prime} 31.36^{\prime \prime} \mathrm{N}\right.$ and $\left.8^{\circ} 14^{\prime} 15.22^{\prime \prime} \mathrm{E}\right)$. The air masses sampled at MPIC represent a mix of urban and rural continental boundary layer air in central Europe (Fröhlich-Nowoisky et al., 2009). A high-volume dichotomous sampler (Solomon et al., 1983) was used to separate and collect coarse and fine aerosol particles on a pair of glass fiber filters. The sampler was operated with a rotary vane pump (Becker VT 4.25) at a total flow rate of approximately $300 \mathrm{~L} \mathrm{~min}^{-1}$, corresponding to a nominal cut-off diameter of $\sim 3 \mu \mathrm{m}$. Coarse particles with aerodynamic diameters larger than the cut-off were collected through a virtual impactor operated in line with the inlet $\left(\sim 27 \mathrm{~L} \mathrm{~min}^{-1}\right)$, and fine particles with aerodynamic diameters smaller than the cut-off were collected from the main gas flow perpendicular to the inlet $\left(\sim 270 \mathrm{~L} \mathrm{~min}^{-1}\right)$.

The sampling period was generally $\sim 7 \mathrm{~d}$, corresponding to a sampled air volume of approximately $3000 \mathrm{~m}^{3}$. A few samples were collected over shorter periods $(1-5 \mathrm{~d}$, $\sim$ $\left.400-2000 \mathrm{~m}^{3}\right)$. A list of all investigated air filter samples (57 coarse and 58 fine particle samples) and of the corresponding sampling times and volumes is given in the online supplement (Tables S1 and S2).

For the investigation of seasonal trends, the air samples were grouped into summer (June, July and August, JJA) (coarse: 18; fine: 18), autumn (September, October and November, SON) (coarse: 14; fine: 14), winter (December, January and February, DJF) (coarse: 12; fine: 13) and spring (March, April and May, MAM) (coarse: 13; fine: 13). Before use, all glass fiber filters were decontaminated by baking at $500{ }^{\circ} \mathrm{C}$ over night. Loaded filters were packed in aluminum foil (also prebaked at $500^{\circ} \mathrm{C}$ ), and stored in a freezer at $-80^{\circ} \mathrm{C}$ until WSOC extraction (Fröhlich-Nowoisky et al., 2009).

\subsection{Sample extraction and analysis (HPLC/MS)}

A filter aliquot (typically $1 / 8$ sector) was transferred into a $7 \mathrm{~mL}$ glass vial with a PTFE-coated cap and extracted with $2 \mathrm{~mL}$ of a water-methanol mixture (methanol volume fraction $10 \%$, water: chromatography grade, Merck, Darmstadt, Germany; methanol: LC-MS grade, Merck, Darmstadt, Germany). The sample vials were sonicated for $30 \mathrm{~min}$ in an ice bath. The extract solution was collected using an Eppendorf pipette with polyethylene (PE) tips. Subsequently, the filters were extracted for the second time following the same 
Table 1. Molar masses, retention time and detection limits of the investigated organic compounds.

\begin{tabular}{|c|c|c|c|c|c|c|c|c|}
\hline Compound & $\begin{array}{l}\mathrm{M}^{\mathrm{a}} \\
\mathrm{g} \mathrm{mol}^{-1}\end{array}$ & $\begin{array}{l}\mathrm{RT}^{\mathrm{b}} \\
\min \end{array}$ & $\begin{array}{l}\mathrm{LOD}^{\mathrm{c}} \\
\mathrm{nmol} \mathrm{L}^{-1}\end{array}$ & $\begin{array}{l}\mathrm{LOQ}^{\mathrm{d}} \\
\mathrm{nmol} \mathrm{L}^{-1}\end{array}$ & $\begin{array}{l}\text { ELOD_coarse } \\
\mathrm{ng} \mathrm{m}^{-3}\end{array}$ & $\begin{array}{l}\text { ELOQ_coarse }^{f} \\
\mathrm{ng} \mathrm{m}^{-3}\end{array}$ & $\begin{array}{l}\text { ELO_fine } \\
\mathrm{ng} \mathrm{m}^{-3}\end{array}$ & $\begin{array}{l}\text { ELOQ_fine } \\
\mathrm{ng} \mathrm{m}^{-3}\end{array}$ \\
\hline Glutaric Acid $\left(\mathrm{C}_{5}\right)$ & 132 & 7.59 & 14.36 & 47.88 & 0.02 & 0.08 & 0.03 & 0.09 \\
\hline Adipic Acid $\left(\mathrm{C}_{6}\right)$ & 146 & 9.01 & 3.01 & 10.03 & 0.01 & 0.02 & 0.01 & 0.02 \\
\hline Pimelic Acid $\left(\mathrm{C}_{7}\right)$ & 160 & 10.35 & 2.69 & 8.96 & 0.01 & 0.02 & 0.01 & 0.02 \\
\hline Suberic Acid $\left(\mathrm{C}_{8}\right)$ & 174 & 11.59 & 19.22 & 64.07 & 0.04 & 0.14 & 0.05 & 0.16 \\
\hline Azelaic Acid $\left(\mathrm{C}_{9}\right)$ & 188 & 12.75 & 19.10 & 63.65 & 0.05 & 0.15 & 0.05 & 0.17 \\
\hline Sebacic Acid $\left(\mathrm{C}_{10}\right)$ & 202 & 13.87 & 1.83 & 6.09 & 0.005 & 0.02 & 0.01 & 0.02 \\
\hline Dodecanedioic Acid $\left(\mathrm{C}_{12}\right)$ & 230 & 15.84 & 1.68 & 5.60 & 0.005 & 0.02 & 0.01 & 0.02 \\
\hline Tridecanedioic Acid $\left(\mathrm{C}_{13}\right)$ & 244 & 17.17 & 1.74 & 5.80 & 0.01 & 0.02 & 0.01 & 0.02 \\
\hline Tetradecanedioic Acid $\left(\mathrm{C}_{14}\right)$ & 258 & 16.77 & 1.62 & 5.39 & 0.01 & 0.02 & 0.01 & 0.02 \\
\hline Hexadecanedioic Acid $\left(\mathrm{C}_{16}\right)$ & 286 & 19.54 & 1.29 & 4.30 & 0.005 & 0.02 & 0.01 & 0.02 \\
\hline Phthalic Acid $(\mathrm{Ph})$ & 166 & 10.72 & 2.85 & 9.50 & 0.01 & 0.02 & 0.01 & 0.02 \\
\hline $\begin{array}{l}\text { 3-Methyl-1,2,3-butanetricarboxylic } \\
\text { acid (3-MBTCA) }\end{array}$ & 204 & 9.45 & 0.59 & 1.98 & 0.002 & 0.01 & 0.002 & 0.01 \\
\hline Pinic Acid & 186 & 11.04 & 1.90 & 6.35 & 0.004 & 0.01 & 0.005 & 0.02 \\
\hline Pinonic Acid (PA) & 184 & 13.88 & 18.06 & 60.20 & 0.04 & 0.14 & 0.05 & 0.16 \\
\hline 4-Nitrocatechol & 155 & 12.30 & 20.10 & 67.00 & 0.04 & 0.13 & 0.04 & 0.15 \\
\hline 2-Nitrophenol & 138 & 14.18 & 17.28 & 57.61 & 0.03 & 0.10 & 0.03 & 0.11 \\
\hline 4-Nitrophenol & 138 & 14.86 & 11.58 & 38.60 & 0.02 & 0.07 & 0.02 & 0.08 \\
\hline
\end{tabular}

a molar mass;

$\mathrm{b}$ retention time;

c limit of detection of the HPLC/MS method defined as 3-fold standard deviation of background signal;

d limit of quantification of the HPLC/MS method defined as 10-fold standard deviation of background signal;

e effective limit of detection for coarse particulate matter (7-day sample);

$\mathrm{f}$ effective limit of quantification for coarse particulate matter (7-day sample);

$\mathrm{g}$ effective limit of detection for fine particulate matter (7-day sample);

$\mathrm{h}$ effective limit of quantification for fine particulate matter (7-day sample).

procedure and the extract solutions were combined and used directly for HPLC/MS analysis (Römpp et al., 2006).

The applied HPLC/MS system consists of a thermostated auto-sampler (Series 200, Perkin Elmer, Norwalk, Connecticut, USA), a degasser and a quaternary pump (1100 Series, Agilent Technologies, Waldbronn, Germany), and a hybrid Qq-TOF mass spectrometer QSTAR (Applied Biosystems MDS SCIEX, Toronto, Canada) with an electrospray ion source (ESI). The ESI source was operated in the negative mode with an ionization voltage of $4 \mathrm{kV}$ at $400^{\circ} \mathrm{C}$. The selected $\mathrm{m} / z$ range was 120 to $300 \mathrm{Da}$. The data processing was performed with the software package Analyst (version QS1.1, Applied Biosystems MDS SCIEX, Toronto, Canada). The room and column temperature were kept at $296 \mathrm{~K}$, and the injection volume was $100 \mu \mathrm{L}$. The chromatographic separation of all investigated compounds was performed with a ReproSil-Pur $\mathrm{C}_{18}$-AQ column $(250 \mathrm{~mm} \times 2.1 \mathrm{~mm}$ I.D., $5 \mu \mathrm{m}$ particle size) in a stainless steel cartridge (Dr. Maisch GmbH, Ammerbuch, Germany) at a flow rate of $400 \mu \mathrm{L} \mathrm{min}^{-1}$. For the first $0.5 \mathrm{~min}$ of each chromatographic run, the composition of the mobile phase was kept at $100 \%$ water with formic acid $(\mathrm{HCOOH}$ volume fraction $0.1 \%$, Chromasolv, Sigma, Seelze, Germany). Then the acetonitrile (ACN, Chromasolv, Sigma, Seelze, Germany) content was increased to $15 \%$ within $3.5 \mathrm{~min}$, and further to $95 \%$ within $16 \mathrm{~min}$. Finally, the mobile phase was reset to initial conditions within $3 \mathrm{~min}$, and the column was equilibrated for 6 min before the next run (Winterhalter et al., 2009). Chromatograms were recorded using the deprotonated molecule signals of the analytes $\left([\mathrm{M}-\mathrm{H}]^{-}\right.$detected at $\left.m / z=\mathrm{M}-1\right)$. The molar masses and retention times of the investigated compounds are listed in Table 1.

The analytes were identified by comparison of retention time and mass spectra with reference standards (Sigma/Fluka, purity $\geq 98 \%$; 3-MBTCA from M. Claeys, University of Antwerp) (Winterhalter et al., 2009). As pure cis-pinic acid was not available, we used the mixture of $c i s$ and trans-pinic acid as reference standard and assumed a response factor of unity between the two isomers for the quantification of $c i s$-pinic acid in aerosol filter samples. Due 
to limited availability and purity of the 3-MBTCA reference standard, we used a calibration curve obtained with 1,3,5-pentatricarboxylic acid and assumed a response factor of unity for the quantification of 3-MBTCA. Small aliphatic dicarboxylic acids consisting of less than five carbon atoms were eluted in the dead volume of the chromatographic column and were thus not investigated in this study.

For every analyte, a linear calibration function of peak area vs. concentration was established and applied for quantification (correlation coefficient $R^{2}>0.98$, number of data points $n=12)$. As detailed in Table 1 , the analytical limits of detection (LOD, $3 \mathrm{~s}$ method, $0.59-20.10 \mathrm{nmol} \mathrm{L}^{-1}$,) and quantification (LOQ, $10 \mathrm{~s}$ method, $1.98-67.00 \mathrm{nmol} \mathrm{L}^{-1}$ ) correspond to effective limits of $0.002-0.05 \mathrm{ng} \mathrm{m}^{-3}$ (ELOD) and $0.01-0.17 \mathrm{ng} \mathrm{m}^{-3}$ (ELOQ) for the detection and quantification of the investigated compounds in the coarse and fine aerosol samples, respectively. Measurement results for blank filter samples were generally below the LOD, except for adipic acid $\left(\mathrm{C}_{6}\right)$ and azelaic acid $\left(\mathrm{C}_{9}\right)$. For these two acids blank values larger than the LOQ, were observed and subtracted from the aerosol sample measurement results. The relative standard deviation of repeated measurements was generally less than $5 \%$. Therefore, the precision of the measurement accounting for imprecision of sample flow control and recovery (Winterhalter et al., 2009) is estimated to be less than $20 \%$.

Mass concentrations of the analytes in the investigated air samples were determined by scaling with the extract volume, filter aliquot fraction, and sampled air volume. Due to the operating principle of the dichotomous sampler, the coarse particle filter samples contained a contribution of fine particles corresponding to the ratio of air flow through the coarse filter to total air flow $(\sim 1 / 10)$. To correct for this interference, $1 / 10$ of the concentration determined for fine particles was subtracted from the concentration determined for coarse particles. All measurement results are listed in the online supplement (Tables S1 and S2). Correlation analyses were performed for all investigated compounds, and the correlation coefficients obtained for coarse and fine particle samples are also listed in the online supplement (Tables S3 and S4). Exponential fits were also performed between concentrations of investigated compounds and inverse temperature, and Arrhenius-type temperature dependencies were found for 3-MBTCA and pinic acid, which is discussed below.

\subsection{Supporting data and model calculations}

Ambient temperature and precipitation data were recorded on top of the 7-story building of the Institute of Atmospheric Physics of the University of Mainz, which is located less than $500 \mathrm{~m}$ away from the sampling site. For correlation analysis, the available temperature data (daily minimum and maximum values) were averaged over the filter sampling period (arithmetic mean values as listed in Tables S1 and S2).
Hydroxyl radical $(\mathrm{OH})$ concentration data were obtained from a simulation (1998-2008) with the ECHAM/MESSy Atmospheric Chemistry model (EMAC, time resolution: $15 \mathrm{~min}$, output frequency: $5 \mathrm{~h}$, averaged over filter sampling period) (Jöckel et al., 2006). The $\mathrm{OH}$ concentration data were used for model calculations investigating the observed Arrhenius-type temperature dependence of 3-MBTCA as detailed in the online supplement and discussed below.

\section{Result and discussion}

\subsection{Concentration levels and seasonal variations}

For a series of linear dicarboxylic acids with 5 to 16 carbon atoms as well as phthalic acid, nitrophenols and pinene oxidation products investigated in this study, the observed concentration ranges, mean values and standard deviations are summarized in Table 2. For comparison we have also performed a comprehensive literature search and compiled data from other atmospheric aerosol studies and locations where these WSOCs have been analyzed.

The mean concentrations of individual dicarboxylic acids in fine particulate matter $\left(\mathrm{PM}_{3}, 0.09-3.76 \mathrm{ng} \mathrm{m}^{-3}\right)$ were generally lower than the values reported from polluted urban areas and megacity regions (Houston, USA; Hong Kong, and 14 economically developed or developing cities in China; $0.29-35.6 \mathrm{ng} \mathrm{m}^{-3}$ ). With regard to total suspended particles (TSP $=$ fine plus coarse particulate matter), the mean concentrations observed in Mainz $\left(0.14-5.71 \mathrm{ng} \mathrm{m}^{-3}\right)$ were higher than values reported from Arctic regions (0.06-0.9 $\mathrm{ng} \mathrm{m}^{-3}$ ), similar to values reported from Philadelphia, USA $\left(\mathrm{PM}_{10}\right.$, $0.5-3.5 \mathrm{ng} \mathrm{m}^{-3}$ ), and generally lower than the values reported from Tokyo, Japan, and Melpitz, Germany ( $\mathrm{PM}_{10}$, n.d. $-25.8 \mathrm{ng} \mathrm{m}^{-3}$ ). The highest mean concentrations in Mainz were observed for phthalic acid $\left(\mathrm{PM}_{3}: 3.76 \mathrm{ng} \mathrm{m}^{-3}\right.$; TSP: $5.71 \mathrm{ng} \mathrm{m}^{-3}$ ) and for the pinene oxidation products pinic acid $\left(\mathrm{PM}_{3}: 1.51 \mathrm{ng} \mathrm{m}^{-3}\right.$; TSP: $\left.2.32 \mathrm{ng} \mathrm{m}^{-3}\right)$ and 3MBTCA ( $\mathrm{PM}_{3}: 5.89 \mathrm{ng} \mathrm{m}^{-3}$; TSP: $\left.6.88 \mathrm{ng} \mathrm{m}^{-3}\right)$.

Seasonal variations of investigated compounds were studied (Figs. 1, 2 and S1), and, to our knowledge, this is the first study reporting a full annual cycle and characteristic differences in the seasonal variation of nitrophenols and 3MBTCA. For most aliphatic dicarboxylic acids the seasonal mean concentration was highest in summer and spring, indicating biogenic or photochemical sources as discussed below (Fig. S1, online supplement). Some of the lower seasonal values found in summer could be attributed to wet deposition of water-soluble compounds due to large amount of precipitation in summer. The total precipitation recorded during the measuring period (2006-2007) amounted to $186.5 \mathrm{~mm}$ in summer, $107.6 \mathrm{~mm}$ in autumn, $136.0 \mathrm{~mm}$ in winter, and $121.6 \mathrm{~mm}$ in spring. 4-Nitrocatechol and 2-nitrophenol also exhibited maximum concentrations in summer, whereas 4nitrophenol exhibited maximum concentrations in winter 
Table 2. Mass concentrations of the investigated compounds in comparison to other studies (range, arithmetic mean and standard deviation).

\begin{tabular}{|c|c|c|c|c|c|}
\hline \multirow[t]{2}{*}{ Compound } & \multicolumn{2}{|c|}{ Concentration $\left(\mathrm{ng} \mathrm{m}^{-3}\right)$} & \multirow{2}{*}{$\begin{array}{l}\text { Particle } \\
\text { size }\end{array}$} & \multirow[t]{2}{*}{ Location, time } & \multirow[t]{2}{*}{ Reference } \\
\hline & Range & Mean $\pm \mathrm{SD}^{\mathrm{a}}$ & & & \\
\hline \multirow[t]{12}{*}{ Glutaric Acid $\left(\mathrm{C}_{5}\right)$} & $0.16-6.15$ & $1.28 \pm 0.99$ & $\mathrm{PM}_{3}^{\mathrm{b}}$ & Mainz, Germany, May 2006-June 2007 & this study \\
\hline & & 30 & $\mathrm{PM}_{2.5}^{\mathrm{c}}$ & 14 cities, China, summer 2003 & Ho et al., 2007 \\
\hline & & 22.1 & $\mathrm{PM}_{2.5}$ & 14 cities, China, winter 2003 & Ho et al., 2007 \\
\hline & & 35.56 & $\mathrm{PM}_{2.5}$ & Hong Kong (urban), summer 2006 & Hu et al., 2008 \\
\hline & & 7.73 & $\mathrm{PM}_{2.5}$ & USA Houston area (suburban), 2000 & Yue and Fraser, 2004 \\
\hline & & 2.3 & $\mathrm{PM}_{10}^{\mathrm{d}}$ & Philadelphia, USA, July-August 1999 & Ray and McDow, 2005 \\
\hline & & 6.8 & $\mathrm{PM}_{10}$ & Melpitz, Germany (rural), 2005 & van Pinxteren and Herrmann, 2007 \\
\hline & $1.24-18.41$ & $2.56 \pm 1.27$ & $\mathrm{TSP}^{\mathrm{e}}$ & Mainz, Germany, May 2006-June 2007 & this study \\
\hline & & 0.9 & TSP & Alert, Arctic, July 1987-June 1988 & Kawamura et al., 1996a \\
\hline & & 11 & TSP & Tokyo, Japan (urban), July 1989 & Kawamura and Yasui, 2005 \\
\hline & & 23.1 & TSP & Tokyo, Japan (urban), June 1989 & Kawamura and Yasui, 2005 \\
\hline & & 18.2 & TSP & Tokyo, Japan (urban), November 1989 & Kawamura and Yasui, 2005 \\
\hline \multirow[t]{12}{*}{ Adipic Acid $\left(\mathrm{C}_{6}\right)$} & $0.17-2.71$ & $0.78 \pm 0.57$ & $\mathrm{PM}_{3}$ & Mainz, Germany, May 2006-June 2007 & this study \\
\hline & & 23.5 & $\mathrm{PM}_{2.5}$ & 14 cities, China, summer 2003 & Ho et al., 2007 \\
\hline & & 15 & $\mathrm{PM}_{2.5}$ & 14 cities, China, winter 2003 & Ho et al., 2007 \\
\hline & & 11.376 & $\mathrm{PM}_{2.5}$ & Hong Kong (urban), summer 2006 & Hu et al., 2008 \\
\hline & & 7.52 & $\mathrm{PM}_{2.5}$ & USA Houston area (suburban), 2000 & Yue and Fraser, 2004 \\
\hline & & 2 & $\mathrm{PM}_{10}$ & Philadelphia, USA, July-August 1999 & Ray and McDow, 2005 \\
\hline & & 13.8 & $\mathrm{PM}_{10}$ & Melpitz, Germany (rural), 2005 & van Pinxteren and Herrmann, 2007 \\
\hline & $0.59-4.69$ & $1.51 \pm 0.84$ & TSP & Mainz, Germany, May 2006-June 2007 & this study \\
\hline & & 0.82 & TSP & Alert, Arctic, July 1987-June 1988 & Kawamura et al., 1996a \\
\hline & & 13 & TSP & Tokyo, Japan (urban), July 1989 & Kawamura and Yasui, 2005 \\
\hline & & 25.8 & TSP & Tokyo, Japan (urban), June 1989 & Kawamura and Yasui, 2005 \\
\hline & & 14.2 & TSP & Tokyo, Japan (urban), November 1989 & Kawamura and Yasui, 2005 \\
\hline \multirow[t]{11}{*}{ Pimelic Acid $\left(\mathrm{C}_{7}\right)$} & $\mathrm{BDL}^{\mathrm{f}}-1.77$ & $0.32 \pm 0.31$ & $\mathrm{PM}_{3}$ & Mainz, Germany, May 2006-June 2007 & this study \\
\hline & & 6.34 & $\mathrm{PM}_{2.5}$ & 14 cities, China, summer 2003 & Ho et al., 2007 \\
\hline & & 6.7 & $\mathrm{PM}_{2.5}$ & 14 cities, China, winter 2003 & Ho et al., 2007 \\
\hline & & 4.564 & $\mathrm{PM}_{2.5}$ & Hong Kong (urban), summer 2006 & Hu et al., 2008 \\
\hline & & 2.58 & $\mathrm{PM}_{2.5}$ & USA Houston area (suburban), 2000 & Yue and Fraser, 2004 \\
\hline & & 1.6 & $\mathrm{PM}_{10}$ & Melpitz, Germany (rural), 2005 & van Pinxteren and Herrmann, 2007 \\
\hline & BDL-2.05 & $0.58 \pm 0.39$ & TSP & Mainz, Germany, May 2006-June 2007 & this study \\
\hline & & 0.13 & TSP & Alert, Arctic, July 1987-June 1988 & Kawamura et al., 1996a \\
\hline & & 4.6 & TSP & Tokyo, Japan (urban), July 1989 & Kawamura and Yasui, 2005 \\
\hline & & 9.9 & TSP & Tokyo, Japan (urban), June 1989 & Kawamura and Yasui, 2005 \\
\hline & & 8.2 & TSP & Tokyo, Japan (urban), November 1989 & Kawamura and Yasui, 2005 \\
\hline \multirow[t]{10}{*}{ Suberic Acid $\left(\mathrm{C}_{8}\right)$} & $0.11-1.87$ & $0.44 \pm 0.35$ & $\mathrm{PM}_{3}$ & Mainz, Germany, May 2006-June 2007 & this study \\
\hline & & 9.06 & $\mathrm{PM}_{2.5}$ & 14 cities, China, summer 2003 & Ho et al., 2007 \\
\hline & & 5.98 & $\mathrm{PM}_{2.5}$ & 14 cities, China, winter 2003 & Ho et al., 2007 \\
\hline & & 5.43 & $\mathrm{PM}_{2.5}$ & USA Houston area (suburban), 2000 & Yue and Fraser, 2004 \\
\hline & & 0.5 & $\mathrm{PM}_{10}$ & Philadelphia, USA, July-August 1999 & Ray and McDow, 2005 \\
\hline & $0.32-2.44$ & $\begin{array}{l}2.1 \\
0.78 \pm 0.45\end{array}$ & $\begin{array}{l}\mathrm{PM}_{10} \\
\mathrm{TSP}\end{array}$ & $\begin{array}{l}\text { Melpitz, Germany (rural), 2005 } \\
\text { Mainz, Germany, May 2006-June } 2007\end{array}$ & $\begin{array}{l}\text { van Pinxteren and Herrmann, } 2007 \\
\text { this study }\end{array}$ \\
\hline & & 0.15 & TSP & Alert, Arctic, July 1987-June 1988 & Kawamura et al., 1996a \\
\hline & & 2.4 & TSP & Tokyo, Japan (urban), July 1989 & Kawamura and Yasui, 2005 \\
\hline & & 11 & TSP & Tokyo, Japan (urban), June 1989 & Kawamura and Yasui, 2005 \\
\hline & & 9.1 & TSP & Tokyo, Japan (urban), November 1989 & Kawamura and Yasui, 2005 \\
\hline
\end{tabular}


Table 2. Continued.

\begin{tabular}{|c|c|c|c|c|c|}
\hline \multirow[t]{2}{*}{ Compound } & \multicolumn{2}{|c|}{ Concentration $\left(\mathrm{ng} \mathrm{m}^{-3}\right)$} & \multirow{2}{*}{$\begin{array}{l}\text { Particle } \\
\text { size }\end{array}$} & \multirow[t]{2}{*}{ Location, time } & \multirow[t]{2}{*}{ Reference } \\
\hline & Range & Mean $\pm \mathrm{SD}^{\mathrm{a}}$ & & & \\
\hline \multirow[t]{11}{*}{ Azelaic Acid $\left(\mathrm{C}_{9}\right)$} & $0.12-4.64$ & $1.60 \pm 1.04$ & $\mathrm{PM}_{3}$ & Mainz, Germany, May 2006-June 2007 & this study \\
\hline & & 31.6 & $\mathrm{PM}_{2.5}$ & 14 cities, China, summer 2003 & Ho et al., 2007 \\
\hline & & 28.9 & $\mathrm{PM}_{2.5}$ & 14 cities, China, winter 2003 & Ho et al., 2007 \\
\hline & & 10.07 & $\mathrm{PM}_{2.5}$ & USA Houston area (suburban), 2000 & Yue and Fraser, 2004 \\
\hline & & 1 & $\mathrm{PM}_{10}$ & Philadelphia, USA, July-August 1999 & Ray and McDow, 2005 \\
\hline & & 2.1 & $\mathrm{PM}_{10}$ & Melpitz, Germany (rural), 2005 & van Pinxteren and Herrmann, 2007 \\
\hline & $0.13-1.45$ & $2.91 \pm 1.25$ & TSP & Mainz, Germany, May 2006-June 2007 & this study \\
\hline & & 0.26 & TSP & Alert, Arctic, July 1987-June 1988 & Kawamura et al., 1996a \\
\hline & & 11 & TSP & Tokyo, Japan (urban), July 1989 & Kawamura and Yasui, 2005 \\
\hline & & 15.1 & TSP & Tokyo, Japan (urban), June 1989 & Kawamura and Yasui, 2005 \\
\hline & & 20.6 & TSP & Tokyo, Japan (urban), November 1989 & Kawamura and Yasui, 2005 \\
\hline \multirow[t]{9}{*}{ Sebacic Acid $\left(\mathrm{C}_{10}\right)$} & $0.06-1.31$ & $0.24 \pm 0.20$ & $\mathrm{PM}_{3}$ & Mainz, Germany, May 2006-June 2007 & this study \\
\hline & & 2.49 & $\mathrm{PM}_{2.5}$ & 14 cities, China, summer 2003 & Ho et al., 2007 \\
\hline & & 4.4 & $\mathrm{PM}_{2.5}$ & 14 cities, China, winter 2003 & Ho et al., 2007 \\
\hline & & 2.58 & $\mathrm{PM}_{2.5}$ & USA Houston area (suburban), 2000 & Yue and Fraser, 2004 \\
\hline & & n.d. ${ }^{g}$ & $\mathrm{PM}_{10}$ & Melpitz, Germany (rural), 2005 & van Pinxteren and Herrmann, 2007 \\
\hline & $0.13-1.45$ & $0.39 \pm 0.24$ & TSP & Mainz, Germany, May 2006-June 2007 & this study \\
\hline & & 1.1 & TSP & Tokyo, Japan (urban), July 1989 & Kawamura and Yasui, 2005 \\
\hline & & 4.9 & TSP & Tokyo, Japan (urban), June 1989 & Kawamura and Yasui, 2005 \\
\hline & & 7.3 & TSP & Tokyo, Japan (urban), November 1989 & Kawamura and Yasui, 2005 \\
\hline \multirow[t]{4}{*}{ Dodecanedioic Acid $\left(\mathrm{C}_{12}\right)$} & BDL-1.24 & $0.09 \pm 0.17$ & $\mathrm{PM}_{3}$ & Mainz, Germany, May 2006-June 2007 & this study \\
\hline & & 0.91 & $\mathrm{PM}_{2.5}$ & 14 cities, China, summer 2003 & Ho et al., 2007 \\
\hline & & 0.29 & $\mathrm{PM}_{2.5}$ & 14 cities, China, winter 2003 & Ho et al., 2007 \\
\hline & BDL-1.34 & $0.15 \pm 0.18$ & TSP & Mainz, Germany, May 2006-June 2007 & this study \\
\hline \multirow[t]{2}{*}{ Tridecanedioic Acid $\left(\mathrm{C}_{13}\right)$} & BDL-2.04 & $0.09 \pm 0.27$ & $\mathrm{PM}_{3}$ & Mainz, Germany, May 2006-June 2007 & this study \\
\hline & BDL-2.11 & $0.14 \pm 0.29$ & TSP & Mainz, Germany, May 2006-June 2007 & this study \\
\hline \multirow[t]{2}{*}{ Tetradecanedioic Acid $\left(\mathrm{C}_{14}\right)$} & BDL-2.05 & $0.11 \pm 0.28$ & $\mathrm{PM}_{3}$ & Mainz, Germany, May 2006-June 2007 & this study \\
\hline & BDL-2.21 & $0.18 \pm 0.31$ & TSP & Mainz, Germany, May 2006-June 2007 & this study \\
\hline \multirow[t]{2}{*}{ Hexadecanedioic Acid $\left(\mathrm{C}_{16}\right)$} & BDL-2.05 & $0.13 \pm 0.29$ & $\mathrm{PM}_{3}$ & Mainz, Germany, May 2006-June 2007 & this study \\
\hline & BDL-2.18 & $0.20 \pm 0.31$ & TSP & Mainz, Germany, May 2006-June 2007 & this study \\
\hline \multirow[t]{5}{*}{ Phthalic Acid (Ph) } & $0.69-13.38$ & $3.76 \pm 2.87$ & $\mathrm{PM}_{3}$ & Mainz, Germany, May 2006-June 2007 & this study \\
\hline & & 3.36 & $\mathrm{PM}_{2.5}$ & Hong Kong (urban), summer 2006 & Hu et al., 2008 \\
\hline & & 12.4 & $\mathrm{PM}_{10}$ & Melpitz, Germany (rural) 2005 & van Pinxteren and Herrmann, 2007 \\
\hline & & 3.5 & $\mathrm{PM}_{10}$ & Philadelphia, USA, July-August 1999 & Ray and McDow, 2005 \\
\hline & $1.24-18.41$ & $5.71 \pm 3.73$ & TSP & Mainz, Germany, May 2006-June 2007 & this study \\
\hline 3-Methyl-1,2,3-butanetricarboxylic acid & $1.6-99.3$ & $13^{\mathrm{h}}$ & $\mathrm{PM}_{1}^{\mathrm{i}}$ & Hyytiälä, Finland, July-August 2005 & Kourtchev et al., 2008a \\
\hline \multirow[t]{9}{*}{ (3-MBTCA) } & $1.9-74$ & 15.8 & $\mathrm{PM}_{2.5}$ & K-puszta, Hungary, summer 2003 & Kourtchev et al., 2009 \\
\hline & & 5.2 & $\mathrm{PM}_{2.5}$ & Jülich, Germany, July 2003 & Kourtchev et al., 2008b \\
\hline & n.d. -1.6 & & $\mathrm{PM}_{2.5}$ & Balbina, Brazil, March-April 1998 & Kubatova et al., $2000^{j}$ \\
\hline & $15.8-130$ & 41.95 & $\mathrm{PM}_{2.5}$ & Research Triangle Park, NC, USA, summer 2003 & Lewandowski et al., $2007^{\mathrm{k}}$ \\
\hline & $12.0-62.9$ & & $\mathrm{PM}_{2.5}$ & SEARCH network, June 2004 & Gao et al., $2006^{1}$ \\
\hline & $0.13-26.00$ & $5.89 \pm 6.15$ & $\mathrm{PM}_{3}$ & Mainz, Germany, May 2006-June 2007 & this study \\
\hline & $0.13-29.72$ & $6.88 \pm 7.04$ & TSP & Mainz, Germany, May 2006-June 2007 & this study \\
\hline & $3.47-25.9$ & & TSP & Mt. Tai, China, May-June 2006 & Fu et al., 2009b \\
\hline & $0.005-2.613$ & $0.647 \pm 0.786$ & TSP & Canadian high Arctic, February-June 1991 & Fu et al., 2009a \\
\hline
\end{tabular}


Table 2. Continued.

\begin{tabular}{|c|c|c|c|c|c|}
\hline \multirow[t]{2}{*}{ Compound } & \multicolumn{2}{|c|}{ Concentration $\left(\mathrm{ng} \mathrm{m}^{-3}\right)$} & \multirow{2}{*}{$\begin{array}{l}\text { Particle } \\
\text { size }\end{array}$} & \multirow[t]{2}{*}{ Location, time } & \multirow[t]{2}{*}{ Reference } \\
\hline & Range & Mean $\pm \mathrm{SD}^{\mathrm{a}}$ & & & \\
\hline \multirow[t]{17}{*}{ Pinic Acid } & $2-29.6$ & $7.7^{\mathrm{h}}$ & $\mathrm{PM}_{1}$ & Hyytiälä, Finland, July-August 2005 & Kourtchev et al., 2008a \\
\hline & $0.6-135$ & 12.2 & $\mathrm{PM}_{2}$ & K-puszta, Hungary, summer 2003 & Kourtchev et al., 2009 \\
\hline & $1.1-21$ & & $\mathrm{PM}_{2.5}$ & Tábua, Portugal, August 1996 & Kavouras et al., 1999b \\
\hline & & 3.0 & $\mathrm{PM}_{2.5}$ & Jülich, Germany, July 2003 & Kourtchev et al., 2008b \\
\hline & $4.5-15.5$ & 9.98 & $\mathrm{PM}_{2.5}$ & Research Triangle Park, NC, USA, summer 2003 & Lewandowski et al., 2007 \\
\hline & $0.38-4.7$ & & $\mathrm{PM}_{2.5}$ & Hyytiälä, Finland, 2001 & Warnke et al., 2006 \\
\hline & $1.1-21$ & & $\mathrm{PM}_{2.5}$ & Hyytiälä, Finland, 2003 & Warnke et al., 2006 \\
\hline & $0.43-3.8$ & & $\mathrm{PM}_{2.5}$ & Jülich, Germany, 2002 & Warnke et al., 2006 \\
\hline & $0.94-12$ & & $\mathrm{PM}_{2.5}$ & Jülich, Germany, 2003 & Warnke et al., 2006 \\
\hline & BDL-9.05 & $1.51 \pm 2.24$ & $\mathrm{PM}_{3}$ & Mainz, Germany, May 2006-June 2007 & this study \\
\hline & $0.06-12.17$ & $2.32 \pm 2.72$ & TSP & Mainz, Germany, May 2006-June 2007 & this study \\
\hline & $0.36-6.27$ & & TSP & Mt. Tai, China, May-June 2006 & Fu et al., 2009b \\
\hline & $0.03-1.357$ & $0.514 \pm 0.401$ & TSP & Canadian high Arctic, February-June 1991 & Fu et al., 2009a \\
\hline & & $2.4 \pm 1.5$ & TSP & Pertouli, Greece, August 1998 & Kavouras and Stephanou, 2002 \\
\hline & $0.4-82.7$ & & TSP & Pertouli, Greece, August 1998 & Kavouras et al., 1999a \\
\hline & $0.48-0.59$ & 0.54 & TSP & Nova Scotia, Canada, July 1996 & Yu et al., 1999b \\
\hline & & 0.5 & TSP & San Bernadino, Canada, September 1998 & Yu et al., 1999b \\
\hline \multirow[t]{12}{*}{ Pinonic Acid (PA) } & $7.1-98$ & & $\mathrm{PM}_{2.5}$ & Tábua, Portugal, August 1996 & Kavouras et al., 1998, 1999b \\
\hline & $0.91-8.2$ & & $\mathrm{PM}_{2.5}$ & Hyytiälä, Finland, 2001 & Warnke et al., 2006 \\
\hline & $0.99-74$ & & $\mathrm{PM}_{2.5}$ & Hyytiälä, Finland, 2003 & Warnke et al., 2006 \\
\hline & $1.1-5.9$ & & $\mathrm{PM}_{2.5}$ & Jülich, Germany, 2002 & Warnke et al., 2006 \\
\hline & $\begin{array}{l}1.4-78 \\
\text { BDL-5.60 }\end{array}$ & $0.60 \pm 1.01$ & $\begin{array}{l}\mathrm{PM}_{2.5} \\
\mathrm{PM}_{3}\end{array}$ & $\begin{array}{l}\text { Jülich, Germany, } 2003 \\
\text { Mainz, Germany, May 2006-June } 2007\end{array}$ & $\begin{array}{l}\text { Warnke et al., } 2006 \\
\text { this study }\end{array}$ \\
\hline & BDL-5.60 & $1.22 \pm 1.33$ & TSP & Mainz, Germany, May 2006-June 2007 & this study \\
\hline & $0.21-21.8$ & & TSP & Mt. Tai, China, May-June 2006 & Fu et al., 2009b \\
\hline & $0.038-0.108$ & $0.069 \pm 0.023$ & TSP & Canadian high Arctic, February-June 1991 & Fu et al., 2009b \\
\hline & & $9.7 \pm 11$ & TSP & Pertouli, Greece, August 1998 & Kavouras and Stephanou, 2002 \\
\hline & $1-25.7$ & & TSP & Pertouli, Greece, August 1998 & Kavouras et al., 1999a \\
\hline & $0.13-0.39$ & 0.26 & TSP & Nova Scotia, Canada, July 1996 & Yu et al., 1999b \\
\hline & & 0.8 & TSP & San Bernadino, Canada, September 1998 & Yu et al., $1999 b$ \\
\hline \multirow[t]{2}{*}{ 4-Nitrocatechol } & BDL-26.30 & $6.40 \pm 17.55$ & $\mathrm{PM}_{3}$ & Mainz, Germany, May 2006-June 2007 & this study \\
\hline & BDL-28.72 & $4.49 \pm 5.32$ & TSP & Mainz, Germany, May 2006-June 2007 & this study \\
\hline \multirow[t]{3}{*}{ 2-Nitrophenol } & BDL-8.51 & $1.57 \pm 1.45$ & $\mathrm{PM}_{3}$ & Mainz, Germany, May 2006-June 2007 & this study \\
\hline & & $3.5 \pm 1.5$ & $\mathrm{PM}_{5}$ & Rome, Italy, Spring, 2003 & Cecinato et al., 2005 \\
\hline & BDL-8.51 & $1.81 \pm 1.70$ & TSP & Mainz, Germany, May 2006-June 2007 & this study \\
\hline \multirow[t]{3}{*}{ 4-Nitrophenol } & BDL-10.22 & $2.48 \pm 2.12$ & $\mathrm{PM}_{3}$ & Mainz, Germany, May 2006-June 2007 & this study \\
\hline & & $17.8 \pm 5.6$ & $\mathrm{PM}_{5}$ & Rome, Italy, Spring 2003 & Cecinato et al., 2005 \\
\hline & BDL-12.52 & $3.78 \pm 2.89$ & TSP & Mainz, Germany, May 2006-June 2007 & this study \\
\hline
\end{tabular}

a standard deviation;

b aerodynamic diameters $<3 \mu \mathrm{m}$ (cut-off of the dichotomous sampler in this study);

c aerodynamic diameters $<2.5 \mu \mathrm{m}$;

$\mathrm{d}$ aerodynamic diameters $<10 \mu \mathrm{m}$;

e total suspended particles (in this study: sum of fine and coarse PM);

${ }^{\mathrm{f}}$ below detection limit (ELOQ, Table 1);

$\mathrm{g}$ not detected;

$\mathrm{h}$ median value;

i aerodynamic diameter $<1 \mu \mathrm{m}$;

j reported as "MW 204";

k reported as "2-hydroxy-4-isopropyladipic acid";

${ }^{1}$ reported as "norpinic diperoxy acid". 
(Fig. 1). The different seasonalities can be attributed to the different sources and formation pathways as discussed below.

The most pronounced seasonal cycles and summertime maxima were observed for the pinene oxidation products 3-MBTCA, pinic acid and pinonic acid (Fig. 2), which exhibited also pronounced Arrhenius-type temperature dependencies that can be attributed to photochemistry and biogenic emissions as discussed below. The average particulate concentration level decreased with increasing volatility from 3-MBTCA (non-volatile) via pinic acid (low/semi-volatile) to pinonic acid (high/semi-volatile, see online supplement).

The concentration of 3-MBTCA was generally higher in the fine particle fraction than in the coarse fraction. During summer and autumn the pinic acid concentration was also higher in the fine fraction than in the coarse fraction, but during winter and spring the concentration was similar in the fine and coarse fractions. The pinonic acid concentration was similar in the fine and coarse fractions during summer and autumns, but during winter and spring the concentration was higher in the coarse fraction. These differences may be related to the different volatilities and concentration levels of the three compounds, to seasonal differences in ambient temperature, and possibly also to seasonal differences in the overall aerosol particle size distribution and composition. Information about the latter parameters is unfortunately not available and further investigations would go beyond the scope of the present study. Nevertheless, we suggest and intend to investigate these effects and interactions in future studies, because they may be relevant for comprehensive characterisation, understanding and modelling of the sources and properties of organic aerosols.

\subsection{Source attribution of dicarboxylic acids and nitrophenols}

Atmospheric photooxidation of volatile and semi-volatile organic compounds is considered to be the main source of dicarboxylic acids in air particulate matter. Mochida et al. (2003a) proposed that small dicarboxylic acids containing up to 7 carbon atoms originate mostly from anthropogenic precursors. Glutaric acid $\left(\mathrm{C}_{5}\right)$ is formed upon oxidation of cyclopentene, cyclohexene and glutardialdehyde (Winterhalter et al., 2009). Adipic acid $\left(\mathrm{C}_{6}\right)$ originates mainly from the oxidation of cyclohexene in the atmosphere (Hatakeyama et al., 1987; Koch et al., 2000), and from the ozonolysis of methylene-cyclohexane and 1-methyl-cyclohexene (Koch et al., 2000). As an aromatic compound, phthalic acid is also considered to originate mostly from anthropogenic sources, including direct emission from automobile, industrial and other anthropogenic sources (manufacturing of plastics, hydrolysis of phthalate esters in the aging of plastics), and the oxidation of polycyclic aromatic hydrocarbons (PAH, Kawamura and Ikushima, 1993; Shiraiwa et al., 2009). The correlation coefficients between phthalic acid and the sum of $\mathrm{C}_{5}$ $\mathrm{C}_{7}$ dicarboxylic acids $\left(R^{2}=0.65\right.$ for coarse particles PM and
0.47 for fine particles) suggest that these compounds originate indeed from related sources.

According to Mochida et al. (2003a), the oxidation of biogenic precursors like unsaturated fatty acids is the main source of $\mathrm{C}_{8}-\mathrm{C}_{10}$ dicarboxylic acids (Kawamura and Gagosian, 1987; Stephanou and Stratigakis, 1993). The most abundant of these compounds was azelaic acid $\left(\mathrm{C}_{9}\right.$, $2.9 \mathrm{ng} \mathrm{m}^{-3}$ in TSP). It originates from the oxidation of unsaturated fatty acids containing a double bond at position 9 (Kawamura and Gagosian, 1987), including oleic acid, which is relatively abundant in air particulate matter and has been studied extensively (Shiraiwa et al., 2010; Zahardis and Petrucci, 2007). The concentrations of suberic acid $\left(\mathrm{C}_{8}, 0.8 \mathrm{ng} \mathrm{m}^{-3}\right.$ in TSP $)$ and sebacic acid $\left(\mathrm{C}_{10}, 0.4 \mathrm{ng} \mathrm{m}^{-3}\right.$ in TSP) were substantially lower than that of azelaic acid. The correlation coefficients suggest that the sources of $\mathrm{C}_{8}$ and $\mathrm{C}_{10}$ are similar $\left(R^{2}=0.71\right.$ and 0.81 for coarse and fine PM), and related but not identical to the sources of $\mathrm{C}_{9}$ $\left(R^{2}=0.36-0.52\right.$, Tables $\mathrm{S} 3$ and $\left.\mathrm{S} 4\right)$.

Long-chain dicarboxylic acids $\left(\mathrm{C}_{12}-\mathrm{C}_{16}\right)$ are generally attributed to the oxidation of $\omega$-hydroxy fatty acids from vascular plants or other biogenic sources (Stephanou and Stratigakis, 1993). The correlation coefficients suggest that the sources of $\mathrm{C}_{12}-\mathrm{C}_{16}$ are more closely related to each other $\left(R^{2}=0.33-0.97\right)$ than to $\mathrm{C}_{8}-\mathrm{C}_{10}\left(R^{2}=0.07-0.75\right)$ or $\mathrm{C}_{9}$ $\left(R^{2}=0.00-0.19\right.$, Tables S3 and S4).

According to Ho et al. (2006), the ratios of adipic acid $\left(\mathrm{C}_{6}\right)$ and phthalic acid $(\mathrm{Ph})$ to azelaic acid $\left(\mathrm{C}_{9}\right)$ can be used to estimate the relative influence of anthropogenic and biogenic sources of organic aerosol. The lower values of the two ratios represent typically biogenic emission; whereas the higher values indicate influence of anthropogenic origin. Comparisons of the two ratios in this study and other location around the world were performed (Table 3 ).

The mean $\mathrm{C}_{6} / \mathrm{C}_{9}$ ratio in $\mathrm{PM}_{3}(0.65$, ranged from 0.21 to 4.35 ) were generally comparable with the values reported from Houston, USA and 14 economically developed or developing cities in China (0.52-0.75). With regard to TSP, the mean ratio observed in Mainz $(0.58$, ranged from 0.23 to 1.81) was generally lower than the other region around the world (TSP: Tokyo, Japan and Arctic, 0.69-3.15; $\mathrm{PM}_{10}$ : Melpitz, Germany and Philadelphia, USA, 2.00-6.57). 84\% of samples in $\mathrm{PM}_{3}$ and $91 \%$ in TSP had the ratio lower than 1.

The mean $\mathrm{Ph} / \mathrm{C}_{9}$ value in $\mathrm{PM}_{3}$ was 3.13 (ranged from 0.76 to 11.78). In TSP, the mean ratio (2.16, ranged from 0.60 to 11.78$)$ was lower than the values in other region $\left(\mathrm{PM}_{10}\right.$, Melpitz, Germany and Philadelphia, USA, 3.50-5.90). The low values of the two ratios indicate that aerosols in Mainz were mainly influenced by biogenic sources.

2-Nitrophenol and 4-nitrophenol can be directly emitted as primary pollutants in combustion exhaust (Tremp et al., 1993), and they can also be formed as secondary pollutants by nitration of phenols in the atmosphere (Atkinson et al., 1992; Dumdei and Obrien, 1984; Grosjean, 1985; Nojima 


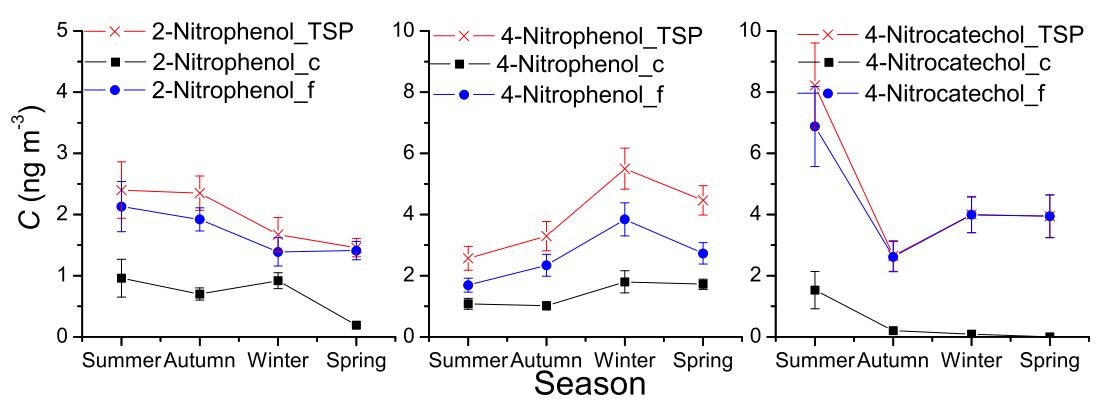

Fig. 1. Seasonal variation of 2-nitrophenol, 4-nitrophenol and 4-nitrocatechol mass concentrations in fine (f), coarse (c), and total particulate matter (TSP). The data points are mean values for different seasons (summer: JJA, autumn: SON, winter: DJF, spring: MAM). The error bars are standard errors of the mean, and the lines are to guide the eye.

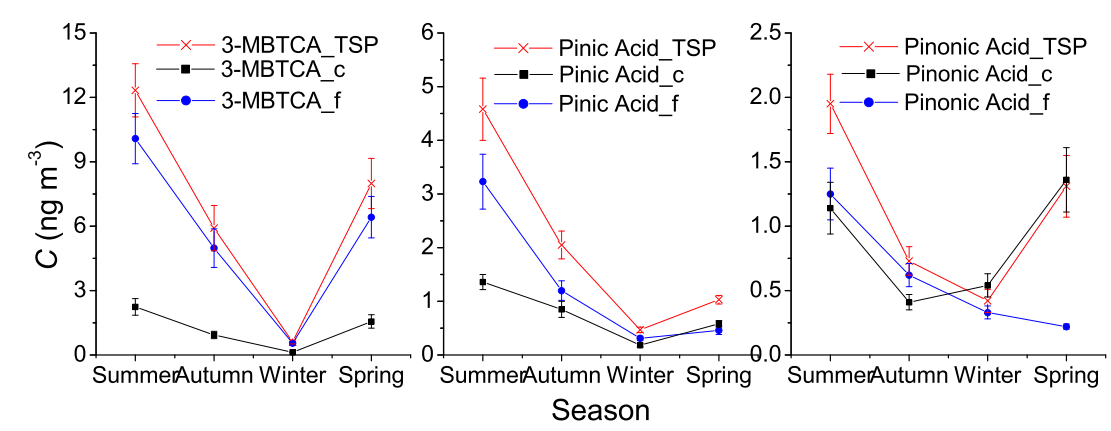

Fig. 2. Seasonal variation of pinene oxidation products: mass concentrations of 3-MBTCA, pinic acid and pinonic acid in fine (f), coarse (c), and total particulate matter (TSP). The data points are mean values for different seasons (summer: JJA, autumn: SON, winter: DJF, spring: MAM). The error bars are standard errors of the mean, and the lines are to guide the eye.

et al., 1975; Olariu et al., 2002). 2-Nitrophenol and 4nitrophenol can originate from the irradiation of benzene$\mathrm{NO}_{\mathrm{x}}$-air mixture (Nojima et al., 1975), whereas only 2nitrophenol can form from various reaction including $\mathrm{OH}-$ initiated reaction in the presence of $\mathrm{NO}_{\mathrm{x}}, \mathrm{NO}_{3}$-initiated reaction and $\mathrm{OH}$-initiated reactions in the presence of $\mathrm{NO}_{3}$ (Atkinson et al., 1992; Olariu et al., 2002). The distinctly different seasonal variations and low correlation coefficients of the two isomers of nitrophenol $\left(R^{2}=0.27\right.$ and 0.33 for coarse and fine PM) indicate that their main sources are different.

The observed summer maximum and correlations with phthalic acid and various aliphatic dicarboxylic acids $\left(R^{2}\right.$ up to 0.59 , Table S4) suggest that atmospheric photochemistry is a major source of 2-nitrophenol. In contrast, the winter maximum of 4-nitrophenol and low correlation coefficients of with dicarboxylic acids $\left(R^{2}<0.32\right.$, Table S4) suggest that primary emissions are more important for this compound than secondary formation. Interestingly, 4-nitrocatechol exhibits a summer maximum like 2-nitrophenol but no correlation with dicarboxylic acids $\left(R^{2}<0.11\right.$, Table S4). These observations indicate primary sources that are enhanced during summer, which could be evaporation from soil as proposed by Gelencser et al. (2002) for humic-like substances (HULIS, Graber and Rudich, 2006). We suggest that further analyses of nitrophenols, nitrocatechol and other nitrated and oxygenated aromatic compounds by HPLC/MS may help to unravel combustion- and soil-related sources of organic particulate matter (Letzel et al., 2001; Schauer et al., 2004).

\subsection{Temperature dependence of pinene oxidation products}

In Fig. 3 the concentrations of 3-MBTCA, pinic acid and pinonic acid observed in the coarse and fine particle fractions and in total plotted against the inverse of average ambient temperature (275-300 K).

Each of the data sets was fitted with an Arrhenius-type expression of the form $X=A \cdot \exp \cdot\left[-E_{\mathrm{a}} /(R \cdot T)\right]$, and the fit parameters are summarized in Table 4 . The results obtained for coarse, fine particulate matter and TSP (fine plus coarse particles) were in overall agreement (Fig. 3), and for simplicity the following discussion is focused on the TSP results.

3-MBTCA exhibited a strong Arrhenius-type temperature dependence with high activation energy and correlation coefficient ( $E_{\mathrm{a}}=126 \pm 10 \mathrm{~kJ} \mathrm{~mol}^{-1}, R^{2}=0.74$, Table 4). Pinic acid also exhibited a pronounced temperature dependence albeit with lower activation energy and correlation coefficient $\left(E_{\mathrm{a}}=84 \pm 9 \mathrm{~kJ} \mathrm{~mol}^{-1}, R^{2}=0.60\right.$, Table 4$)$, whereas the temperature dependence of pinonic acid was very weak 
Table 3. Ratios between the mass concentrations of adipic acid, phthalic acid and azelaic acid $\left(\mathrm{C}_{6} / \mathrm{C}_{9}\right.$ and $\left.\mathrm{Ph} / \mathrm{C}_{9}\right)$ in comparison to other studies.

\begin{tabular}{llllll}
\hline Location & Time & $\mathrm{C}_{6} / \mathrm{C}_{9}$ & $\mathrm{Ph} / \mathrm{C}_{9}$ & $\begin{array}{l}\text { Particle } \\
\text { size }\end{array}$ & Reference \\
\hline Mainz, Germany & June 2006-May 2007 & 0.65 & 3.13 & $\mathrm{PM}_{3}$ & this study \\
14 cities, China & 2003 & $0.52-0.74$ & & $\mathrm{PM}_{2.5}$ & Ho et al., 2007 \\
Houston area, USA & 2000 & 0.75 & & $\mathrm{PM}_{2.5}$ & Yue and Fraser, 2004 \\
Melpitz, Germany & 2005 & 6.57 & 5.90 & $\mathrm{PM}_{10}$ & van Pinxteren and Herrmann, 2007 \\
Philadelphia, USA & July-August 1999 & 2.00 & 3.50 & $\mathrm{PM}_{10}$ & Ray and McDow, 2005 \\
Mainz, Germany & June 2006-May 2007 & 0.58 & 2.16 & $\mathrm{TSP}$ & this study \\
Alert, Arctic & July 1987-June 1988 & 3.15 & & $\mathrm{TSP}$ & Kawamura et al., 1996a \\
Tokyo, Japan & 1989 & $0.69-1.71$ & & $\mathrm{TSP}$ & Kawamura and Yasui, 2005 \\
\hline
\end{tabular}

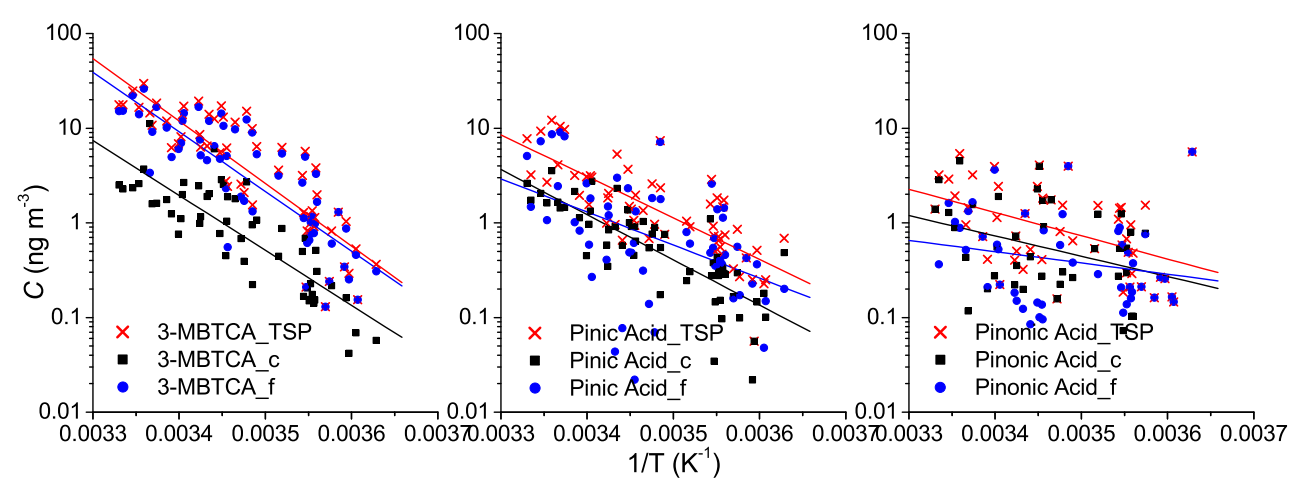

Fig. 3. Arrhenius-type temperature dependencies observed for pinene oxidation products (275-300 K): mass concentrations of 3-MBTCA, pinic acid and pinonic acid in fine (f), coarse (c) and total particulate matter (TSP) plotted against inverse temperature. The data points represent individual samples, the lines are exponential fits, and the corresponding Arrhenius parameters are listed in Table 4.

$\left(E_{\mathrm{a}}=47 \pm 13 \mathrm{~kJ} \mathrm{~mol}^{-1}, R^{2}=0.21\right.$, Table 4$)$. In order to explain the observed temperature dependencies and differences, we considered the following steps involved in the atmospheric oxidation of pinene and formation of pinic acid, pinonic acid and 3-MBTCA: (1) emission of pinene; (2) oxidation of pinene and formation of pinic and pinonic acid; (3) oxidation of pinonic acid and formation of 3-MBTCA.

As detailed in the online supplement and illustrated in Table 4 , the temperature dependence of the pinene emission rate can be described by an Arrhenius activation energy of $\sim 76 \mathrm{~kJ} \mathrm{~mol}^{-1}$ (Komenda et al., 2003).

The oxidation rate of pinene depends primarily on the concentration of oxidants (ozone, $\mathrm{OH}$, etc.) and on rate coefficients of the involved gas phase reactions. For typical atmospheric conditions and oxidant concentration levels, however, the lifetime of pinene is relatively short $(\sim 5 \mathrm{~h}$ for $\alpha$-pinene and $\sim 3.5 \mathrm{~h}$ for $\beta$-pinene, $k(\mathrm{OH})=5.3 \times 10^{-11} \mathrm{~cm}^{3} \mathrm{~s}^{-1}$ for $\alpha$-pinene and $k(\mathrm{OH})=7.9 \times 10^{-11} \mathrm{~cm}^{3} \mathrm{~s}^{-1}$ for $\beta$-pinene (Atkinson et al.,
2006) and typical average $C_{\mathrm{OH}}=1 \times 10^{6} \mathrm{~cm}^{-3}$ ) compared to the transport, mixing and averaging times involved in the collection of air samples in this study (of the order of one week). Thus, we assume that the oxidation of pinene is not rate-limiting and that the temperature dependence of the formation of pinic and pinonic acid can be approximated by the temperature dependence of pinene emission. The plausibility of this assumption is confirmed by the agreement between the observed temperature dependence of pinic acid concentration and the calculated temperature dependence of pinene emission (Table 4).

The oxidation rate of pinonic acid depends primarily on gas-particle partitioning, on the concentration of $\mathrm{OH}$ radicals, and on the $\mathrm{OH}$ reaction rate coefficient (Jimenez et al., 2009; Hallquist et al., 2009). As detailed in the online supplement and illustrated in Fig. 4, the temperature dependence of gas-particle partitioning of pinonic acid is very weak and can be approximated by an Arrhenius activation energy of $\sim 0.21 \mathrm{~kJ} \mathrm{~mol}^{-1}$ (Table 4 ). Moreover, pinonic acid 
Table 4. Arrhenius parameters for observed and modeled temperature dependencies (275-300 K): pinene oxidation product mass concentrations (3-MBTCA, pinic acid, pinonic acid) in fine, coarse and total particulate matter (TSP) as shown in Fig. 3; emission of pinene, gas-particle partitioning of pinonic acid, and concentration of $\mathrm{OH}$ radicals as described in the online supplement and shown in Fig. 4.

\begin{tabular}{lllll}
\hline & $A^{\mathrm{a}}$ & $E_{\mathrm{a}}\left(\mathrm{kJ} \mathrm{mol}^{-1}\right)^{\mathrm{b}}$ & $R^{2 \mathrm{c}}$ & $n^{\mathrm{d}}$ \\
\hline 3-MBTCA (TSP) & $3.29 \times 10^{23} \mathrm{ng} \mathrm{m}^{-3}$ & $126 \pm 10$ & 0.74 & 52 \\
(fine) & $2.41 \times 10^{22} \mathrm{ng} \mathrm{m}^{-3}$ & $121 \pm 11$ & 0.69 & 58 \\
(coarse) & $1.05 \times 10^{20} \mathrm{ng} \mathrm{m}^{-3}$ & $111 \pm 10$ & 0.72 & 52 \\
Pinonic acid (TSP) & $2.82 \times 10^{8} \mathrm{ng} \mathrm{m}^{-3}$ & $46.9 \pm 12.7$ & 0.21 & 35 \\
(fine) & $5.49 \times 10^{3} \mathrm{ng} \mathrm{m}^{-3}$ & $22.8 \pm 15.0$ & 0.05 & 36 \\
(coarse) & $1.45 \times 10^{7} \mathrm{ng} \mathrm{m}^{-3}$ & $41.1 \pm 18.3$ & 0.12 & 35 \\
Pinic acid (TSP) & $2.54 \times 10^{15} \mathrm{ng} \mathrm{m}^{-3}$ & $83.9 \pm 9.1$ & 0.60 & 56 \\
(fine) & $9.78 \times 1^{11} \mathrm{ng} \mathrm{m}^{-3}$ & $66.8 \pm 16.3$ & 0.24 & 56 \\
(coarse) & $2.05 \times 10^{16} \mathrm{ng} \mathrm{m}^{-3}$ & $91.3 \pm 8.9$ & 0.66 & 57 \\
Pinene emission & $6.31 \times 10^{14} \mathrm{ng} \mathrm{m}^{-3}$ & $75.6 \pm 3.3$ & & \\
Pinonic acid gas-particle- & 1.09 & $0.213 \pm 0.011$ & 0.95 & 27 \\
partitioning & & & & \\
OH concentration & $4.52 \times 10^{24} \mathrm{~cm}^{-3}$ & $110 \pm 8$ & 0.79 & 59 \\
\hline
\end{tabular}

a pre-exponential factor (Fig. 3 and online supplement);

$\mathrm{b}$ activation energy (Fig. 3 and online supplement);

c correlation coefficient;

$\mathrm{d}$ number of data points.

is expected to reside almost exclusively in the gas phase (>99\%, online supplement) under the conditions relevant for this study (Müller et al., 2010), and thus gas-particle partitioning should have little influence on the observed temperature dependencies.

As detailed in the online supplement, the $E_{\mathrm{a}}$ of $\mathrm{OH}$ reaction rate coefficient cause insignificant temperature dependence of overall $\mathrm{OH}$ oxidation of pinonic acid.

The atmospheric concentration of $\mathrm{OH}$ radicals depends on a wide range of processes, including the photolysis of ozone by solar UV radiation (Crutzen et al., 1999; Rohrer and Berresheim, 2006). Due to radiative heating, the intensity of solar radiation at a given location usually varies in conjunction with diurnal and seasonal variations of temperature. To explore the effective dependence of $\mathrm{OH}$ radical concentration on ambient temperature at our sampling location, we used model data from the ECHAM/MESSy Atmospheric Chemistry Model (EMAC, Jöckel et al., 2006). As detailed in the online supplement, these data could be fitted with an Arrhenius-type expression $\left(R^{2}=0.79\right)$ and with an apparent Arrhenius activation energy of $110 \pm 8 \mathrm{~kJ} \mathrm{~mol}^{-1}$.

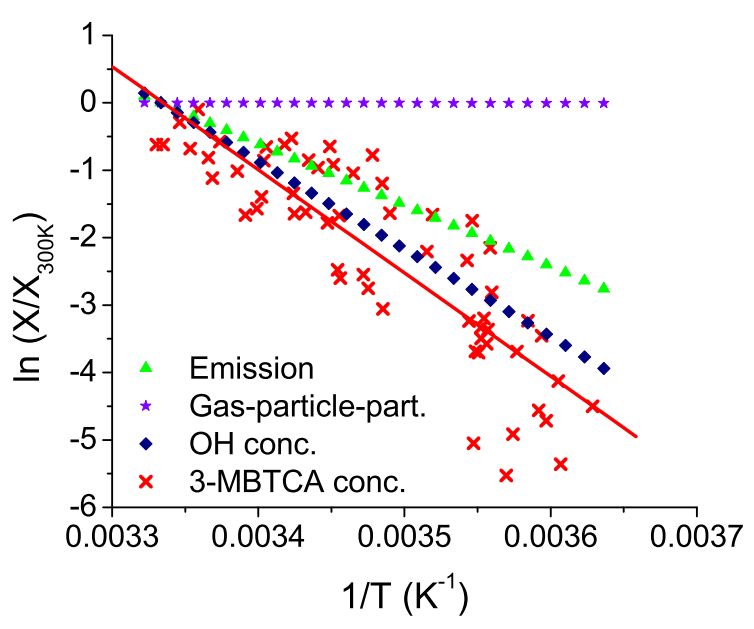

Fig. 4. Normalized Arrhenius-type plot of the temperature dependencies $(275-300 \mathrm{~K})$ observed for the concentration of 3-MBTCA $\left(C_{3}\right.$-MBTCA,TSP, data points and solid line) and modeled for the emission of pinene $\left(C_{\mathrm{PE}}\right)$, the gas-particle partitioning of pinonic acid (FGP $\mathrm{FA})$, and the concentration of $\mathrm{OH}$ radicals $\left(C_{\mathrm{OH}}\right.$, dotted lines). For each parameter, $X_{300 \mathrm{~K}}$ is the fit value at $300 \mathrm{~K}$. The model assumptions and calculations are described in the online supplement. 
As illustrated in Fig. 4, the model-derived effective temperature dependence of $\mathrm{OH}$ radical concentration is similar to the observed temperature dependence of 3-MBTCA concentration and shows only $16 \mathrm{~kJ} \mathrm{~mol}^{-1}$ difference, which is within the error range. The agreement suggests that the $\mathrm{OH}-$ initiated oxidation of pinonic acid is indeed the rate-limiting step in the formation of 3-MBTCA, because the temperature dependence of a process consisting of multiple steps is generally dominated by the temperature dependence of the rate-limiting step. None of the other temperature dependencies considered above and illustrated in Fig. 4 appears strong enough to explain the observed temperature dependence of 3-MBTCA, and it appears plausible that the formation and concentration of 3-MBTCA is limited by the OH-initiated oxidation of pinonic acid. The result is also consistent with regard to the very weak temperature dependence observed for the pinonic acid concentration, which may result from an effective cancelation of the temperature dependencies of the formation from pinene emission and oxidation (in analogy to pinic acid) and the loss by $\mathrm{OH}$ oxidation. Reaction rate coefficient $k(\mathrm{OH})$ is larger for pinonic acid $(1.04 \times$ $\left.10^{-11} \mathrm{~cm}^{3} \mathrm{~s}^{-1}\right)$ than for pinic acid $\left(8.7 \times 10^{-12} \mathrm{~cm}^{3} \mathrm{~s}^{-1}\right)$ at $298 \mathrm{~K}$ (Vereecken and Peeters, 2002), resulting in an OH lifetime of $\sim 27 \mathrm{~h}$ for pinonic acid and $\sim 32 \mathrm{~h}$ for pinic acid, using a typical average $\mathrm{OH}$ concentration of $1 \times 10^{6}$ radical $\mathrm{cm}^{-3}$. It appears not very likely that potential temperature dependencies of other relevant processes, like dry and wet deposition of the investigated compounds, would be more important and better suited to explain the observations.

Nevertheless, the above considerations have to be regarded as a first approximation and simple conceptual model approach to explain the observations. A full mechanistic understanding and quantification will require detailed numerical model studies including potential effects of atmospheric transport and deposition. This would go beyond the scope of the present study, but we suggest and intend to pursue such investigations in follow-up studies. Similarity of $E_{\mathrm{a}}$ for observed Arrhenius concentration and model $\mathrm{OH}$ concentration suggests that the $\mathrm{OH}$-initiated oxidation is the rate-limiting step of 3-MBTCA formation. The difference of $16 \mathrm{~kJ} \mathrm{~mol}^{-1}$ is within the range of uncertainties and might also be related to sink processes (chemical loss, dry/wet deposition).

\section{Conclusions}

Based on the results of this study we suggest that further analyses of nitrated and oxygenated aromatic compounds by HPLC/MS may help to unravel combustion- and soilrelated sources of organic particulate matter in comparison to primary biological aerosols containing fatty acids and SOA formed from gaseous biogenic precursors like pinene.

To our knowledge, this is the first study to explain field observations of pinene oxidation products (pinic acid, pinonic acid, 3-MBTCA) with a model analysis of the tem- perature dependencies of emissions, gas-particle partitioning and chemical reactions. The model calculations suggest that the $\mathrm{OH}$ radical concentration and the $\mathrm{OH}$-initiated oxidation of pinonic acid limit the formation and explain the observed Arrhenius-type temperature dependence of 3MBTCA, whereas the influence of gas-particle partitioning appears negligible. Thus, 3-MBTCA appears to be a suitable molecular tracer for the chemical aging of biogenic SOA by $\mathrm{OH}$ radicals.

\section{Supplementary material related to this article is available online at: http://www.atmos-chem-phys.net/10/7859/2010/ acp-10-7859-2010-supplement.pdf.}

Acknowledgements. This study has been supported by the Max Planck Society (MPG), the International Max Planck Research School for Atmospheric Chemistry and Physics (IMPRS), the Geocycles Cluster in Mainz (LEC Rheinland-Pfalz), and the European integrated project of aerosol, cloud, climate, and air quality interactions (EUCAARI). The authors would like to thank J. Fröhlich for providing filter samples and related information, M. Claeys for providing synthetic 3-methyl-1,2,3-butanetricarboxylic acid standards for HPLC/MS analysis, and S. C. Lai and H. Yang for helpful suggestions and support. P. Jöckel and the members of the "Earth System Modelling Group of the Max Planck Institute for Chemistry" are gratefully acknowledged for providing $\mathrm{OH}$ concentration data from the EMAC model.

The service charges for this open access publication have been covered by the Max Planck Society.

Edited by: V.-M. Kerminen

\section{References}

Atkinson, R., Aschmann, S. M., and Arey, J.: Reactions of $\mathrm{OH}$ and $\mathrm{NO}_{3}$ radicals with phenol, cresols, and 2-nitrophenol at 296 K \pm 2 K, Environ. Sci. Technol., 26, 1397-1403, 1992.

Atkinson, R. and Arey, J.: Gas-phase tropospheric chemistry of biogenic volatile organic compounds: a review, Atmos. Environ., 37, S197-S219, 10.1016/s1352-2310(03)00391-1, 2003.

Atkinson, R., Baulch, D. L., Cox, R. A., Crowley, J. N., Hampson, R. F., Hynes, R. G., Jenkin, M. E., Rossi, M. J., Troe, J., and IUPAC Subcommittee: Evaluated kinetic and photochemical data for atmospheric chemistry: Volume II - gas phase reactions of organic species, Atmos. Chem. Phys., 6, 3625-4055, doi:10.5194/acp-6-3625-2006, 2006.

Belloli, R., Barletta, B., Bolzacchini, E., Meinardi, S., Orlandi, M., and Rindone, B.: Determination of toxic nitrophenols in the atmosphere by high-performance liquid chromatography, J. Chromatogr. A, 846, 277-281, 1999.

Cecinato, A., Di Palo, V., Pomata, D., Sciano, M. C. T., and Possanzini, M.: Measurement of phase-distributed nitrophenols in Rome ambient air, Chemosphere, 59, 679-683, doi:10.1016/j.chemosphere.2004.10.045, 2005. 
Crutzen, P. J., Lawrence, M. G., and Pöschl, U.: On the background photochemistry of tropospheric ozone, Tellus A, 51, 123-146, 1999.

Dumdei, B. E. and Obrien, R. J.: Toluene degradation products in simulated atmospheric conditions, Nature, 311, 248-250, 1984.

Fröhlich-Nowoisky, J., Pickersgill, D. A., Despres, V. R., and Pöschl, U.: High diversity of fungi in air particulate matter, Proceedings of the National Academy of Sciences of the United States of America, 106, 12814-12819, 10.1073/pnas.0811003106, 2009.

Fu, P., Kawamura, K., Chen, J., and Barrie, L. A.: Isoprene, monoterpene, and sesquiterpene oxidation products in the high Arctic aerosols during late winter to early summer, Environ. Sci. Technol., 43, 4022-4028, 2009a.

Fu, P. Q., Kawamura, K., Pochanart, P., Tanimoto, H., Kanaya, Y., and Wang, Z. F.: Summertime contributions of isoprene, monoterpenes, and sesquiterpene oxidation to the formation of secondary organic aerosol in the troposphere over Mt. Tai, Central East China during MTX2006, Atmos. Chem. Phys. Discuss., 9, 16941-16972, doi:10.5194/acpd-9-16941-2009, 2009b.

Gao, S., Surratt, J. D., Knipping, E. M., Edgerton, E. S., Shahgholi, M., and Seinfeld, J. H.: Characterization of polar organic components in fine aerosols in the southeastern United States: identity, origin, and evolution, J. Geophys. Res.-Atmos., 111, D14314, doi:10.1029/2005jd006601, 2006.

Gelencser, A., Hoffer, A., Krivacsy, Z., Kiss, G., Molnar, A., and Meszaros, E.: On the possible origin of humic matter in fine continental aerosol, J. Geophys. Res.-Atmos., 107, 4137, doi:10.1029/2001jd001299, 2002.

Graber, E. R. and Rudich, Y.: Atmospheric HULIS: How humiclike are they? A comprehensive and critical review, Atmos. Chem. Phys., 6, 729-753, doi:10.5194/acp-6-729-2006, 2006.

Grosjean, D.: Reactions of o-cresol and nitrocresol with $\mathrm{NO}_{\mathrm{X}}$ in sunlight and with ozone nitrogen dioxide mixtures in the dark, Environ. Sci. Technol., 19, 968-974, 1985.

Hallquist, M., Wenger, J. C., Baltensperger, U., Rudich, Y., Simpson, D., Claeys, M., Dommen, J., Donahue, N. M., George, C., Goldstein, A. H., Hamilton, J. F., Herrmann, H., Hoffmann, T., Iinuma, Y., Jang, M., Jenkin, M. E., Jimenez, J. L., Kiendler-Scharr, A., Maenhaut, W., McFiggans, G., Mentel, Th. F., Monod, A., Prévôt, A. S. H., Seinfeld, J. H., Surratt, J. D., Szmigielski, R., and Wildt, J.: The formation, properties and impact of secondary organic aerosol: current and emerging issues, Atmos. Chem. Phys., 9, 5155-5236, doi:10.5194/acp-95155-2009, 2009.

Hatakeyama, S., Ohno, M., Weng, J. H., Takagi, H., and Akimoto, H.: Mechanism for the formation of gaseous and particulate products from ozone-cycloalkene reactions in air, Environ. Sci. Technol., 21, 52-57, 1987.

Hatakeyama, S., Izumi, K., Fukuyama, T., and Akimoto, H.: Reactions of ozone with alpha-pinene and beta-pinene in air - yields of gaseous and particulate products, J. Geophys. Res.-Atmos., 94, 13013-13024, 1989.

Hatakeyama, S., Izumi, K., Fukuyama, T., Akimoto, H., and Washida, N.: Reactions of $\mathrm{OH}$ with alpha-pinene and betapinene in air - estimate of global CO production from the atmospheric oxidation of terpenes, J. Geophys. Res.-Atmos., 96, 947-958, 1991.

Herterich, R.: Gas chromatographic determination of nitrophenols in atmospheric liquid water and airborne-particulates, J. Chromatogr., 549, 313-324, 1991.

Ho, K. F., Lee, S. C., Cao, J. J., Kawamura, K., Watanabe, T., Cheng, Y., and Chow, J. C.: Dicarboxylic acids, ketocarboxylic acids and dicarbonyls in the urban roadside area of Hong Kong, Atmos. Environ., 40, 3030-3040, 2006.

Ho, K. F., Cao, J. J., Lee, S. C., Kawamura, K., Zhang, R. J., Chow, J. C., and Watson, J. G.: Dicarboxylic acids, ketocarboxylic acids, and dicarbonyls in the urban atmosphere of China, J. Geophys. Res.-Atmos., 112, D22S27, 10.1029/2006JD008011, 2007.

Hoffmann, T., Odum, J. R., Bowman, F., Collins, D., Klockow, D., Flagan, R. C., and Seinfeld, J. H.: Formation of organic aerosols from the oxidation of biogenic hydrocarbons, J. Atmos. Chem., 26, 189-222, 1997.

Hu, D., Bian, Q., Li, T. W. Y., Lau, A. K. H., and Yu, J. Z.: Contributions of isoprene, monoterpenes, beta-caryophyllene, and toluene to secondary organic aerosols in Hong Kong during the summer of 2006, J. Geophys. Res.-Atmos., 113, D22206, 10.1029/2008JD010437, 2008.

Jaoui, M., Kleindienst, T. E., Lewandowski, M., Offenberg, J. H., and Edney, E. O.: Identification and quantification of aerosol polar oxygenated compounds bearing carboxylic or hydroxyl groups. 2. Organic tracer compounds from monoterpenes, Environ. Sci. Technol., 39, 5661-5673, doi:10.1021/Es048111b, 2005 .

Jöckel, P., Tost, H., Pozzer, A., Brühl, C., Buchholz, J., Ganzeveld, L., Hoor, P., Kerkweg, A., Lawrence, M. G., Sander, R., Steil, B., Stiller, G., Tanarhte, M., Taraborrelli, D., van Aardenne, J., and Lelieveld, J.: The atmospheric chemistry general circulation model ECHAM5/MESSy1: consistent simulation of ozone from the surface to the mesosphere, Atmos. Chem. Phys., 6, 50675104, doi:10.5194/acp-6-5067-2006, 2006.

Kavouras, I. G., Mihalopoulos, N., and Stephanou, E. G.: Formation of atmospheric particles from organic acids produced by forests, Nature, 395, 683-686, 1998.

Kavouras, I. G., Lawrence, J., Koutrakis, P., Stephanou, E. G., and Oyola, P.: Measurement of particulate aliphatic and polynuclear aromatic hydrocarbons in Santiago de Chile: source reconciliation and evaluation of sampling artifacts, Atmos. Environ., 33, 4977-4986, 1999a.

Kavouras, I. G., Mihalopoulos, N., and Stephanou, E. G.: Secondary organic aerosol formation vs primary organic aerosol emission: In situ evidence for the chemical coupling between monoterpene acidic photooxidation products and new particle formation over forests, Environ. Sci. Technol., 33, 1028-1037, 1999b.

Kavouras, I. G. and Stephanou, E. G.: Direct evidence of atmospheric secondary organic aerosol formation in forest atmosphere through heteromolecular nucleation, Environ. Sci. Technol., 36, 5083-5091, 2002.

Kawamura, K. and Gagosian, R. B.: Implications of omegaoxocarboxylic acids in the remote marine atmosphere for photooxidation of unsaturated fatty-acids, Nature, 325, 330-332, 1987.

Kawamura, K. and Kaplan, I. R.: Motor exhaust emissions as a primary source for dicarboxylic-acids in Los-Angeles ambient air, Environ. Sci. Technol., 21, 105-110, 1987.

Kawamura, K. and Ikushima, K.: Seasonal changes in the distribu- 
tion of dicarboxylic acids in the urban atmosphere, Environ. Sci. Technol., 27, 2227-2235, 1993.

Kawamura, K. and Usukura, K.: Distributions of low molecular weight dicarboxylic acids in the North Pacific aerosol samples, J. Oceanogr., 49, 271-283, 1993.

Kawamura, K., Kasukabe, H., and Barrie, L. A.: Source and reaction pathways of dicarboxylic acids, ketoacids and dicarbonyls in arctic aerosols: one year of observations, Atmos. Environ., 30, 1709-1722, 1996a.

Kawamura, K., Semere, R., Imai, Y., Fujii, Y., and Hayashi, M.: Water soluble dicarboxylic acids and related compounds in Antarctic aerosols, J. Geophys. Res.-Atmos., 101, 18721-18728, 1996b.

Kawamura, K. and Yasui, O.: Diurnal changes in the distribution of dicarboxylic acids, ketocarboxylic acids and dicarbonyls in the urban Tokyo atmosphere, Atmos. Environ., 39, 1945-1960, 2005.

Koch, S., Winterhalter, R., Uherek, E., Kolloff, A., Neeb, P., and Moortgat, G. K.: Formation of new particles in the gas-phase ozonolysis of monoterpenes, Atmos. Environ., 34, 4031-4042, 2000.

Komenda, M., Kobel, K., Koppmann, R., and Wildt, J.: Comparability of biogenic VOC emission rate measurements under laboratory and ambient conditions at the example of monoterpene emissions from Scots pine (Pinus sylvestris), J. Atmos. Chem., 45, 1-23, 2003.

Kourtchev, I., Ruuskanen, T. M., Keronen, P., Sogacheva, L., Dal Maso, M., Reissell, A., Chi, X., Vermeylen, R., Kulmala, M., Maenhaut, W., and Claeys, M.: Determination of isoprene and alpha-/beta-pinene oxidation products in boreal forest aerosols from Hyytiala, Finland: diel variations and possible link with particle formation events, Plant. Biol., 10, 138-149, 2008a.

Kourtchev, I., Warnke, J., Maenhaut, W., Hoffmann, T., and Claeys, M.: Polar organic marker compounds in $\mathrm{PM}_{2.5}$ aerosol from a mixed forest site in western Germany, Chemosphere, 73, 13081314, 2008b.

Kourtchev, I., Copolovici, L., Claeys, M., and Maenhaut, W.: Characterization of atmospheric aerosols at a forested site in central europe, Environ. Sci. Technol., 43, 4665-4671, 2009.

Kubatova, A., Vermeylen, R., Claeys, M., Cafmeyer, J., Maenhaut, W., Roberts, G., and Artaxo, P.: Carbonaceous aerosol characterization in the Amazon basin, Brazil: novel dicarboxylic acids and related compounds, Atmos. Environ., 34, 5037-5051, 2000.

Kubatova, A., Vermeylen, R., Claeys, M., Cafmeyer, J., and Maenhaut, W.: Organic compounds in urban aerosols from Gent, Belgium: characterization, sources, and seasonal differences, J. Geophys. Res.-Atmos., 107, 8343, doi:10.1029/2001JD000556, 2002.

Kundu, S., Kawamura, K., Andreae, T. W., Hoffer, A., and Andreae, M. O.: Molecular distributions of dicarboxylic acids, ketocarboxylic acids and alpha-dicarbonyls in biomass burning aerosols: implications for photochemical production and degradation in smoke layers, Atmos. Chem. Phys., 10, 2209-2225, doi:10.5194/acp-10-2209-2010, 2010.

Lefer, B. L., Talbot, R. W., Harriss, R. C., Bradshaw, J. D., Sandholm, S. T., Olson, J. O., Sachse, G. W., Collins, J., Shipham, M. A., Blake, D. R., Klemm, K. I., Klemm, O., Gorzelska, K., and Barrick, J.: Enhancement of acidic gases in biomass burning impacted air masses over Canada, J. Geophys. Res.-Atmos., 99,
1721-1737, 1994.

Legrand, M. and DeAngelis, M.: Light carboxylic acids in Greenland ice: a record of past forest fires and vegetation emissions from the boreal zone, J. Geophys. Res.-Atmos., 101, 4129-4145, 1996.

Letzel, T., Pöschl, U., Wissiack, R., Rosenberg, E., Grasserbauer, M., and Niessner, R.: Phenyl-modified reversed-phase liquid chromatography coupled to atmospheric pressure chemical ionization mass spectrometry: A universal method for the analysis of partially oxidized aromatic hydrocarbons, Anal. Chem., 73, 1634-1645, doi:10.1021/ac001079t, 2001.

Leuenberger, C., Ligocki, M. P., and Pankow, J. F.: Trace organiccompounds in rain .4. Identities, concentrations, and scavenging mechanisms for phenols in urban air and rain, Environ. Sci. Technol., 19, 1053-1058, 1985.

Lewandowski, M., Jaoui, M., Kleindienst, T. E., Offenberg, J. H., and Edney, E. O.: Composition of $\mathrm{PM}_{2.5}$ during the summer of 2003 in Research Triangle Park, North Carolina, Atmos. Environ., 41, 4073-4083, 2007.

Luttke, J. and Levsen, K.: Occurrence and formation of nitrated phenols in clouds at two European sites, Proceedings of Eurotrac Symposium ' 96 - Transport and transformation of pollutants in the troposphere, 1, 101-107, 1997.

Luttke, J., Scheer, V., Levsen, K., Wunsch, G., Cape, J. N., Hargreaves, K. J., StoretonWest, R. L., Acker, K., Wieprecht, W., and Jones, B.: Occurrence and formation of nitrated phenols in and out of cloud, Atmos. Environ., 31, 2637-2648, 1997.

Müller, L., Reinnig, M. C., Naumann, K. H., Saathoff, H., Donahue, N., and Hoffmann, T.: Formation of 3-methyl-1,2,3butanetricarboxylic acid via gas phase $\mathrm{OH}$-oxidation of pinonic acid - a mass spectrometric study of SOA aging, Atmos. Chem. Phys. Discuss., in preparation, 2010.

Mochida, M., Kawabata, A., Kawamura, K., Hatsushika, H., and Yamazaki, K.: Seasonal variation and origins of dicarboxylic acids in the marine atmosphere over the western North Pacific, J. Geophys. Res.-Atmos., 108, 4193, doi:10.1029/2002JD002355, 2003a.

Mochida, M., Umemoto, N., Kawamura, K., and Uematsu, M.: Bimodal size distribution of $\mathrm{C}_{2}-\mathrm{C}_{4}$ dicarboxylic acids in the marine aerosols, Geophys. Res. Lett., 30, 1672, doi:10.1029/2003GL017451, 2003b.

Narukawa, M., Kawamura, K., Takeuchi, N., and Nakajima, T.: Distribution of dicarboxylic acids and carbon isotopic compositions in aerosols from 1997 Indonesian forest fires, Geophys. Res. Lett., 26, 3101-3104, 1999.

Nojima, K., Kawaguchi, A., Ohya, T., Kanno, S., and Hirobe, M.: Studies on photochemical-reaction of air-pollutants .10. Identification of nitrophenols in suspended particulates, Chem. Pharm. Bull., 31, 1047-1051, 1983.

Nojima, M., Nagata, T., and Tokura, N.: Studies on photochemistry of aromatic hydrocarbons, Chemosphere, 4, 77-82, 1975.

Olariu, R. I., Klotz, B., Barnes, I., Becker, K. H., and Mocanu, R.: FT-IR study of the ring-retaining products from the reaction of $\mathrm{OH}$ radicals with phenol, o-, m-, and p-cresol, Atmos. Environ., 36, 3685-3697, PiiS1352-2310(02)00202-9, 2002.

Pavuluri, C. M., Kawamura, K., and Swaminathan, T.: Watersoluble organic carbon, dicarboxylic acids, ketoacids, and alphadicarbonyls in the tropical Indian aerosols, J. Geophys. Res.Atmos., 115, D11302, doi:10.1029/2009jd012661, 2010. 
Pöschl, U.: Atmospheric aerosols: composition, transformation, climate and health effects, Angew. Chem. Int. Edit., 44, 75207540, doi:10.1002/anie.200501122, 2005.

Ray, J. and McDow, S. R.: Dicarboxylic acid concentration trends and sampling artifacts, Atmos. Environ., 39, 7906-7919, 2005.

Rohrer, F. and Berresheim, H.: Strong correlation between levels of tropospheric hydroxyl radicals and solar ultraviolet radiation, Nature, 442, 184-187, 2006.

Römpp, A., Winterhalter, R., and Moortgat, G. K.: Oxodicarboxylic acids in atmospheric aerosol particles, Atmos. Environ., 40, 6846-6862, 2006.

Schauer, C., Niessner, R., and Pöschl, U.: Analysis of nitrated polycyclic aromatic hydrocarbons by liquid chromatography with fluorescence and mass spectrometry detection: air particulate matter, soot, and reaction product studies, Anal. Bioanal. Chem., 378, 725-736, doi:10.1007/s00216-003-2449-1, 2004.

Shafer, W. E. and Schonherr, J.: Accumulation and transport of phenol, 2-nitrophenol, and 4-nitrophenol in plant cuticles, Ecotox. Environ. Safe., 10, 239-252, 1985.

Shiraiwa, M., Garland, R. M., and Pöschl, U.: Kinetic double-layer model of aerosol surface chemistry and gas-particle interactions (K2-SURF): Degradation of polycyclic aromatic hydrocarbons exposed to $\mathrm{O}_{3}, \mathrm{NO}_{2}, \mathrm{H}_{2} \mathrm{O}, \mathrm{OH}$ and $\mathrm{NO} 3$, Atmos. Chem. Phys., 9, 9571-9586, doi:10.5194/acp-9-9571-2009, 2009.

Shiraiwa, M., Pfrang, C., and Pöschl, U.: Kinetic multi-layer model of aerosol surface and bulk chemistry (KM-SUB): the influence of interfacial transport and bulk diffusion on the oxidation of oleic acid by ozone, Atmos. Chem. Phys., 10, 3673-3691, doi:10.5194/acp-10-3673-2010, 2010.

Solomon, P. A., Moyers, J. L., and Fletcher, R. A.: High-volume dichotomous virtual Impactor for the fractionation and collection of particles according to aerodynamic size, Aerosol Sci. Techn., 2, 455-464, 1983.

Stephanou, E. G. and Stratigakis, N.: Oxocarboxylic and alpha,omega-dicarboxylic acids - photooxidation products of biogenic unsaturated fatty-acids present in urban aerosols, Environ. Sci. Technol., 27, 1403-1407, 1993.

Szmigielski, R., Surratt, J. D., Gomez-Gonzalez, Y., Van der Veken, P., Kourtchev, I., Vermeylen, R., Blockhuys, F., Jaoui, M., Kleindienst, T. E., Lewandowski, M., Offenberg, J. H., Edney, E. O., Seinfeld, J. H., Maenhaut, W., and Claeys, M.: 3-Methyl1,2,3-butanetricarboxylic acid: An atmospheric tracer for terpene secondary organic aerosol, Geophys. Res. Lett., 34, L24811, doi:10.1029/2007GL031338, 2007.
Tremp, J., Mattrel, P., Fingler, S., and Giger, W.: Phenols and nitrophenols as tropospheric pollutants - emissions from automobile exhausts and phase-transfer in the atmosphere, Water Air Soil Poll., 68, 113-123, 1993.

van Pinxteren, D. and Herrmann, H.: Determination of functionalised carboxylic acids in atmospheric particles and cloud water using capillary electrophoresis/mass spectrometry, J. Chromatogr. A, 1171, 112-123, 2007.

Vereecken, L. and Peeters, J.: Enhanced H-atom abstraction from pinonaldehyde, pinonic acid, pinic acid, and related compounds: theoretical study of C-H bond strengths, Phys. Chem. Phys. Chem., 4, 467-472, 2002.

Warnke, J., Bandur, R., and Hoffmann, T.: Capillary-HPLC-ESIMS/MS method for the determination of acidic products from the oxidation of monoterpenes in atmospheric aerosol samples, Anal. Bioanal. Chem., 385, 34-45, doi:10.1007/s00216-0060340-6, 2006.

Winterhalter, R., Kippenberger, M., Williams, J., Fries, E., Sieg, K., and Moortgat, G. K.: Concentrations of higher dicarboxylic acids $\mathrm{C}_{5} \mathrm{C}_{13}$ in fresh snow samples collected at the High Alpine Research Station Jungfraujoch during CLACE 5 and 6, Atmos. Chem. Phys., 9, 2097-2112, doi:10.5194/acp-9-2097-2009, 2009.

Yu, J. Z., Cocker, D. R., Griffin, R. J., Flagan, R. C., and Seinfeld, J. H.: Gas-phase ozone oxidation of monoterpenes: gaseous and particulate products, J. Atmos. Chem., 34, 207-258, 1999a.

Yu, J. Z., Griffin, R. J., Cocker, D. R., Flagan, R. C., Seinfeld, J. H., and Blanchard, P.: Observation of gaseous and particulate products of monoterpene oxidation in forest atmospheres, Geophys. Res. Lett., 26, 1145-1148, 1999b.

Yue, Z. W. and Fraser, M. P.: Polar organic compounds measured in fine particulate matter during TexAQS 2000, Atmos. Environ., 38, 3253-3261, 2004.

Zahardis, J. and Petrucci, G. A.: The oleic acid-ozone heterogeneous reaction system: products, kinetics, secondary chemistry, and atmospheric implications of a model system - a review, Atmos. Chem. Phys., 7, 1237-1274, doi:10.5194/acp-7-1237-2007, 2007. 